\title{
Vibration Control and Dynamical Model of a Thermal-Electrical-Mechanical Coupled Smart Cantilevered Beam
}

\author{
Ting Zhang* ${ }^{* 1}$, H.G. Li ${ }^{2}$, J.J. Zhao ${ }^{3}$ \\ 1, 2, 3 State Key Lab. of vibration, Shock \& Noise, Shanghai Jiao Tong University, Shanghai, 200240, \\ People's Republic of China \\ ${ }^{1}$ ztwcl@sjtu.edu.cn \\ ${ }^{2}$ hgli@sjtu.edu.cn \\ ${ }^{3}$ snowstorm0316@163.com
}

\begin{abstract}
Keywords: Cantilever, Vibration Control, Piezoelectric, Finite Element, Adaptive Pole Placement Control
\end{abstract}

\begin{abstract}
Piezoelectric actuators used in vibration control and high precision control have been known widely in recent years. Especially in aeronautics and MEMS systems, their use is spread from vibration suppression to position control. In this paper, a finite element model (FEM) of a piezoelectric actuator and cantilever in thermal environment is presented to suppress vibration effectively. In other words, the finite element model is namely thermal-electrical-mechanical coupled FEM. Based on a 8-node plane finite element, the modal analysis, the harmonic analysis and the transient analysis have been obtained in the current work. Therefore a transfer function model will be attained through the harmonic analysis by identification method in order to control vibration by control law. In addition, the controller will be designed with the adaptive pole placement control (APPC). Finally, through simulation, the thermal influence is considerable for natural frequencies, harmonic response and free vibration. Moreover, the APPC is a significant plan to vibration control in the paper.
\end{abstract}

\section{Introduction}

Applications of smart materials, such as piezoelectric materials, magnetostrictive materials, magneto-rheological fluids, electrorheological fluids and shape memory alloys, have been developed increasingly in recent years. Piezoelectric materials can be the most popular smart materials in actuators applications by reason of low power consumption, low weight, and ease in bonding with the structures. In [1], Meng, G., L. Ye, et al. present a scheme involving dynamic modeling of a smart structure, designing laws and closed-loop simulation in a finite element environment. Actually, the piezoelectric materials and the structures will expand with heat and contract with cold, the piezoelectric materials will produce voltage when the temperature value is difference in both ends and the piezoelectric materials will generate heat when applied high voltage. In [2], this paper presents the dynamic analysis of the influence of the coupling between thermal, electrical and mechanical fields for different modes; in [3], in this paper a finite element model is developed for the active control of thermally induced vibration of laminated composite shells with piezoelectric sensors and actuators.

There are many methods to model the system's dynamical model. First, the theory method is common in many researches. The finite element model is mainstream means for mechanical systems in modern world. It can be derived using Hamilton's principle and Lagrange variation theory. in [4], the system equations can be derived using Hamilton's principle and the assumed mode method; There is other modeling way: identification modeling, in [5], this study presents results of multimodal vibration suppression of a smart flexible cantilever beam with piezoceramic actuator and sensor by suing a pole placement controller; in [6], V. SETHI and G. SONG put forward subspace identification after acquiring a model frame. Moreover the control laws for vibration suppression vary from one application aim to another. in [7], this paper presents the design and the experimental implementation of fast and periodic output feedback controllers to minimize 
structural vibration, using collocated piezoelectric actuators and sensors; in [8], the vibration suppression and precision positioning capabilities of the adaptive feedforward control have been discussed; in [9], Park, J. K., G. Washington, et al. adopt the Generalized predictive control to vibration reduction.

In this paper, the authors introduce a thermal-electrical-mechanical coupled model using plane finite element formulations and pole placement control law in the twice part. The simulation result of modal analysis, harmonical analysis, free vibration and vibration control will be introduced in the third part. Finally, the conclusion and outlook will be derived from simulation result.

\section{Thermal-electrical-mechanical coupled finite element model}

Constitutive linear equations. In the present study, a cantilevered beam structure bonded with a piezoelectric actuator is shown in Fig.1. Elastic field, electric field and thermal field for a typical intellectual structure are considered to be linearly coupled.

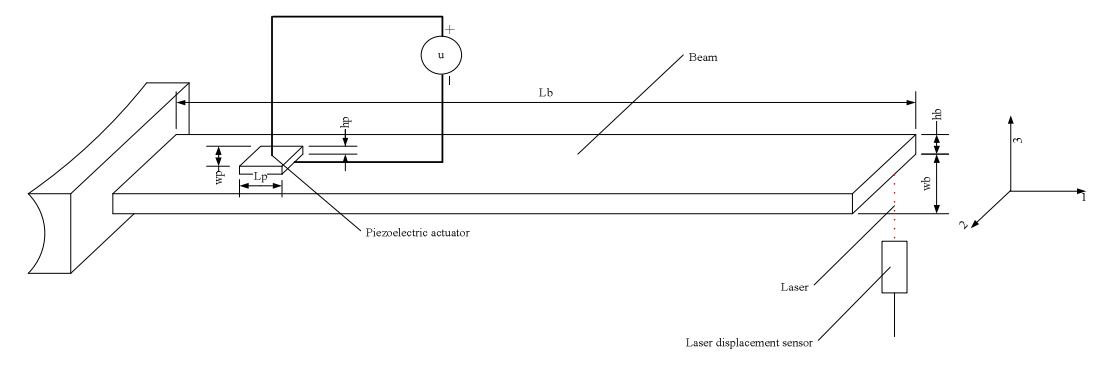

Fig. 1. A cantilevered beam bonded with a piezoelectric actuator

In Ansys v13, the element PLANE233 has Structural-Thermal and thermal-piezoelectric capabilities which we are most interested in. The element input data includes eight nodes and structural, thermal, and electrical material properties. And the element has eight nodes with up to four degrees of freedom per node $\left\{u_{1}, u_{3}, V, T\right\}$. Here, $u_{x}$ and $u_{z}$ are the displacement in $x$ and $z$ directions, $V$ is the applied voltage, and $T$ is the temperature rise from the stress free reference temperature. Accordingly, the strain and stress equations should be written as equation (1). If we consider the thermal influence for structure and piezoelectric actuator, A thermal-electricalmechanical coupled formulation (equations (2)) is introduced in order to represent direct and converse piezoelectric effect.

$$
\begin{aligned}
& \left\{\begin{array}{l}
x_{1}=\frac{\partial u_{x}}{\partial x}-z \frac{\partial \psi_{x}}{\partial x} \\
X_{1}=c_{11} x_{1} \\
\psi_{x}(x, z, t)=\frac{\partial u_{y}(x, z, t)}{\partial x}
\end{array}\right. \\
& \left\{\begin{array}{l}
X_{1}=c_{11}^{D} x_{1}-h_{31} D_{3}-\lambda_{1}^{X} \theta \\
E_{3}=-h_{31} x_{1}+\beta_{33}^{x} D_{3}+p_{3}^{E} \theta \\
S=-\lambda_{1}^{S} x_{1}+p_{3}^{S} D_{3}+\alpha \theta
\end{array}\right.
\end{aligned}
$$

where $x, X$ and $c$ represent the mechanical stress, the mechanical strain and the elastic stiffness constant respectively. $s, d, E, D$ and $\varepsilon$ represent elastic compliance constant, piezoelectric stress constant, electric field, electric displacement and free dielectric constant respectively. $h$ and $\beta$ represent piezoelectric stiffness constant and free dielectric isolation rate respectively. $\lambda, \theta, p$, $S$ and $\alpha$ represent the coefficient of thermal expansion, the temperature, the pyroelectric contant, the entropy of the system and the ratio of the heat capacity and the strain free temperature. 
Finite element model. The dynamical model of a smart structure is developed under the following three assumptions: there is no any relative motion in the bonding between the beam and the piezoelectric actuator; the piezoelectric actuator is $\mathrm{z}-\mathrm{x}$ cutted; the optical axis of the piezoelectric actuator is the thickness direction of beam.

The transversal displacement of the beam can be expressed as equation (3),

$$
\begin{aligned}
& u_{z}(x, t)=\left[N_{1}, N_{2}, N_{3}, \cdots \cdots, N_{n}\right]\left[q_{1}, q_{2}, q_{3}, \cdots \cdots, q_{n}\right]^{T}=N q \\
& x_{1}=H L N q=B q
\end{aligned}
$$

where $q$ is generalized nodal coordinates vector and $N$ is shape functions vector. $B=H L N, H$ is the matrix that stores the truncated constants matrix from Equation (1), $L$ is the matrix where all of the displacement field relations are truncated.

Kinetic energy terms, the elastic, electrical and thermal energy terms and the virtual work term are expressed as equations (5) (6) and (7) respectively. And the finite element formulation is equation (8).

$$
\begin{aligned}
& E_{k}=\frac{1}{2} \dot{q}^{T}\left(\int_{0}^{h_{e} \int_{0}^{l_{e}}} \rho_{e} w_{e} N^{T} N d x d z\right) \dot{q}=\frac{1}{2} \dot{q} M^{e} \dot{q} \\
& \delta E_{p}=\int_{V_{e}}\left(X_{1} \delta x_{1}+E_{3} \delta D_{3}+S \delta \theta\right) d V_{e}=\int_{V_{e}}\left(c_{11} x_{1} \delta x_{1}+\varepsilon D_{3} \delta D_{3}+\alpha \theta \delta \theta\right) d V_{e} \\
& =\delta\left(q^{T} K_{q q}^{e} q+\phi K_{\phi q}^{e} q+\theta K_{\theta q}^{e} q+\phi K_{\phi \phi}^{e} \phi+\theta K_{\theta \phi}^{e} \phi+\theta K_{\theta \theta}^{e} \theta\right) \\
& \delta W=\int_{0}^{l_{e}} f \delta u_{z} d x-\int_{0}^{l_{e}} c \dot{u}_{z} \delta u_{z} d x+\int_{0}^{l_{e}} \frac{V}{h_{e}} \delta \frac{\phi}{A_{x}} d x+\int_{V_{e}} Q \delta \theta d V_{e} \\
& =R_{q}^{e} \delta q+R_{\phi}^{e} \delta \phi+R_{\theta}^{e} \delta \theta \\
& \delta J(q, \phi, \theta)=\delta \int_{t_{0}}^{t_{f}} L(q, \phi, \theta ; t) d t=\int_{t_{0}}^{t_{f}}\left(\delta E_{k}-\delta E_{p}+\delta W\right) d t=0
\end{aligned}
$$

where $\rho_{e}$ and $w_{e}$ are the density and width of the element respectively. $f$ is the external disturbance, $c$ is the structure damping, $V$ is the voltage across the structure, and $Q$ is the heat flux, $h_{e}$ and $v_{e}$ are thickness and volume of the smart structure element respectively.

Element mass damp and stiffness matrices for the system can be written as shown by using Eqs. (1)-(8), then $M=\sum M^{e}, C=\sum C^{e}, K=\sum K^{e}, R=\sum R^{e}$. The equation of motion for a system with a single piezoelectric patch can be constructed:

$$
\left[\begin{array}{ccc}
M & 0 & 0 \\
0 & L & 0 \\
0 & 0 & 0
\end{array}\right]\left[\begin{array}{c}
\ddot{q} \\
\ddot{\phi} \\
\ddot{\theta}
\end{array}\right]+\left[\begin{array}{ccc}
C & 0 & 0 \\
0 & R & 0 \\
0 & 0 & C_{t}
\end{array}\right]\left[\begin{array}{c}
\dot{q} \\
\dot{\phi} \\
\dot{\theta}
\end{array}\right]+\left[\begin{array}{ccc}
K_{u u} & K_{u \phi} & K_{u \theta} \\
K_{\phi u} & K_{\phi \phi} & K_{\phi \theta} \\
K_{\theta u} & K_{\theta \phi} & K_{\theta \theta}
\end{array}\right]\left[\begin{array}{l}
q \\
\phi \\
\theta
\end{array}\right]=\left[\begin{array}{l}
R_{q} \\
R_{\phi} \\
R_{\theta}
\end{array}\right]
$$

\section{The Adaptive Pole Placement Control Law}

As is known that the dynamic response can be described as a continuous time state space representation (equation (10)). The feedback controller $u$ can be determined by Equation (11) when the state feedback gain matrix $K$ is obtained by feedback law and desired poles. Substituting Equation (11) into Equation (12) and considering the reference input, the closed loop system is written as equation (12).

$$
\begin{aligned}
& \left\{\begin{array}{l}
\dot{x}=A x+B u \\
y=C x+D u
\end{array}\right. \\
& u=v-K x \\
& \left\{\begin{array}{l}
\dot{x}=(A-B K) x+B v \\
y=C x
\end{array}\right.
\end{aligned}
$$

where $x$ and $y$ are the state variable vector and the output tip displacement of the smart beam respectively. $A, B, C$ and $D$ present the state matrix, the input matrix, the output matrix and direct transfer matrix respectively. Here $D$ is zeros matrix. $v$ is reference input. 
The matrix $A-B K$ determines the eigenvalues of the close loop system (Equation (12)) by desired poles in the Ackermann's method. However, the full states $x$ may not be easily achieved in practical situations. Then a state observer must be designed to obtain the entire states of the system. The design of the state observer can be done by the above pole assignment. The dynamical former of the observer is written as:

$$
\dot{\hat{x}}=(A-G C) \hat{x}+G y+B u
$$

where $\mathrm{G}$ is observing matrix and is obtained by pole placent technique.

The observer's state $\hat{x}$ will approach asymptotically to the actual state $x$ when the eigenvalues of the matrix $A-B K$ are negative. The temperature of the external environment is always changing, and the thermal-electrical-mechanical model of the systems is always time-varying. We must identify the system model and the controller all the time to main the control precision. Accordingly, the adaptive pole placement control system with a observer is attained by Equation (12) and Equation (13), shown in Fig. 2.

\section{Results and discussion}

The smart beam system works at very rugged environment in temperature from $-40^{\circ}$ to $+150^{\circ}$. There will be thermal-mechanical coupled energy transformation for the beam and the piezoelectric actuator. Moreover, there will be electric-mechanical coupled energy transformation for the piezoelectric actuator so as to actuate the beam when the piezoelectric patch is applied $100 \mathrm{~V}$ voltage.

The harmonic analysis. The results of harmonic analysis in Ansys v13 is shown in figure 3. From the results, we see that the natural frequencies are different with the different conditions. In adiabat environment, the natural frequencies in structural conditions are almost in structural-electric conditions. However, in thermal environment, the natural frequencies are increasing with the temperature increasing.
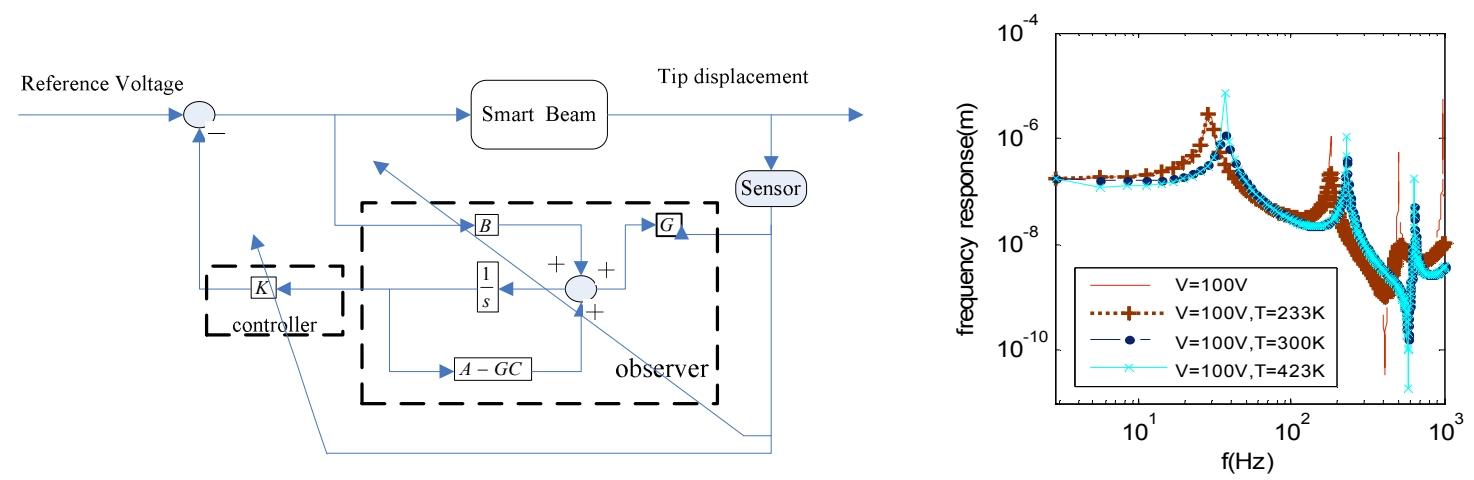

Fig.2.The state feedback system with a observer Fig.3.Harmonic analysis in different conditions

Vibration control results. Figure 4, 5, 6 and 7 show the vibration control results in mechanical-electrical conditions, mechanical- electrical-thermal (223K) conditions, mechanical-electrical-thermal $(300 \mathrm{~K})$ conditions and mechanical- electrical-thermal (523K) conditions respectively. Fig. 4, 5, 6 and 7 (a) shows the identification results, the identifiable curve can fit very well the harmonic data in mechanical-electrical conditions. And Fig. 4, 5, 6, and 7 (b) shows the free vibration results, square wave response and frequency response with pole placement controllers and without controllers respectively. The blue lines represent the curves without controllers and the red lines represent the curves with pole placement controllers. From the simulation results, the APPC is a effective control method to vibration control. 


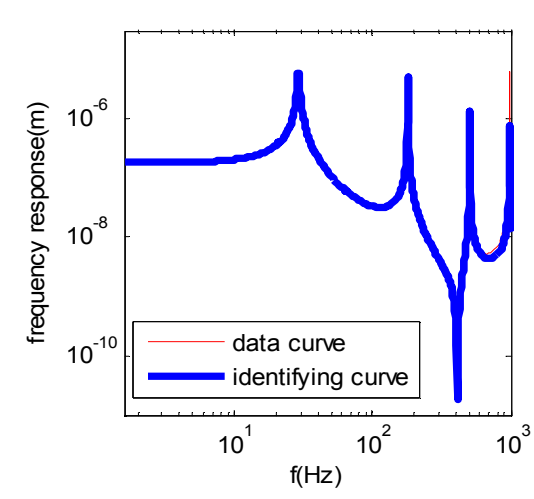

(a)

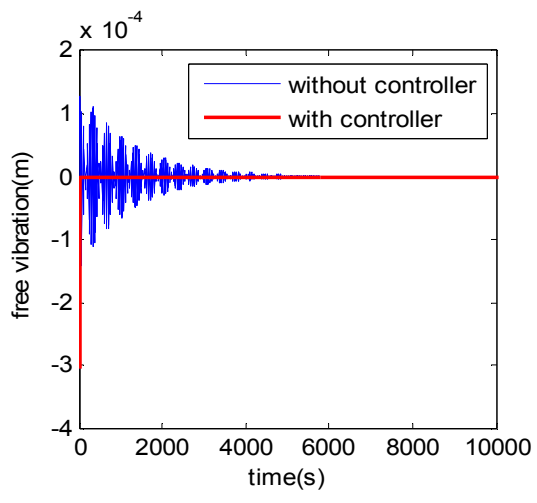

(b)

Fig. 4. The results in mechanical-electrical conditons (a) frequence response ---identification curve - experimental curve (b) free vibration - without controller - with controller

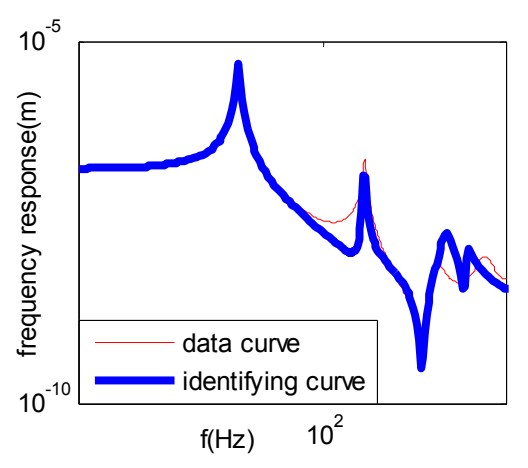

(a)

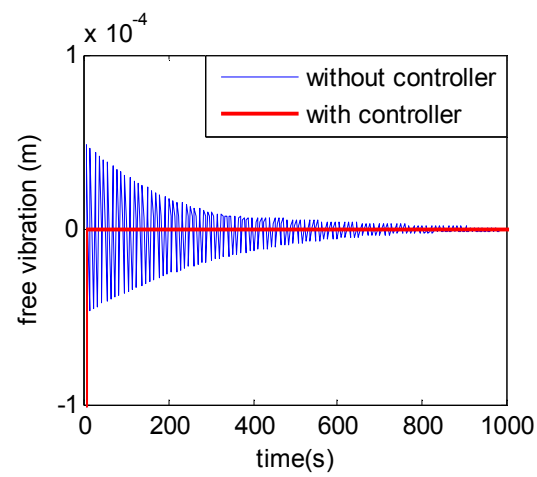

(b)

Fig. 5. The results in mechanical-electrical-thermal (223K) conditions (a) frequence response ---identification curve — experimental curve (b) free vibration — without controller — with controller

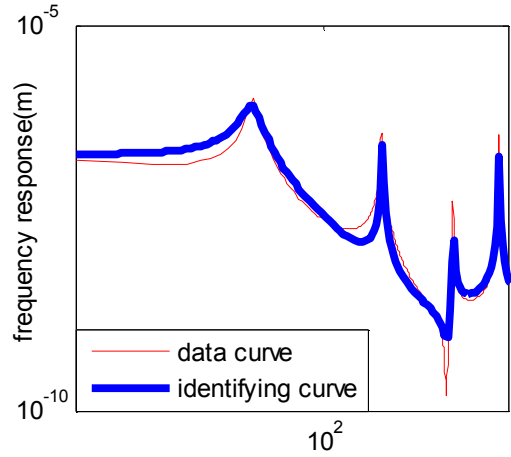

(a)

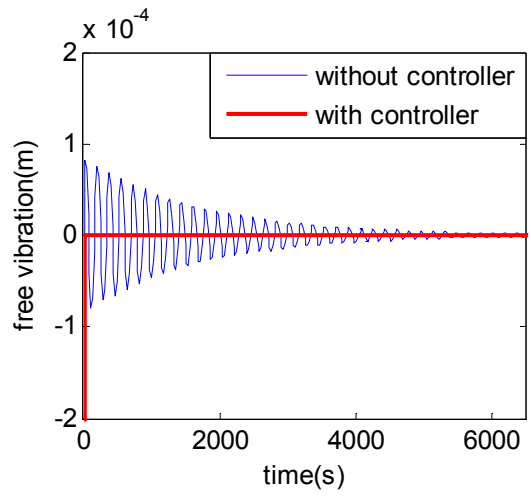

(b)

Fig. 6. The results in mechanical-electrical-thermal (223K) conditions (a) frequence response ---identification curve - experimental curve (b) free vibration — without controller — with controller

\section{Conclusion and Outlook}

From above mechanical-electrical-thermal finite element theory, we can see the mechanicalelectrical-thermal FEM can tell clearly transformation of the mechanical energy, electrical energy and thermal energy. The simulation results prove APPC is a effective control for vibration control. 
In this paper, we analyze the dynamical properties of mechanical-electrical-thermal FEM in linear model. Actually, the piezoelectric actuators have some nonlinear characteristics, such as hystersis, saturation and so on. Furthermore, the controlled structures have nonlinear properties.

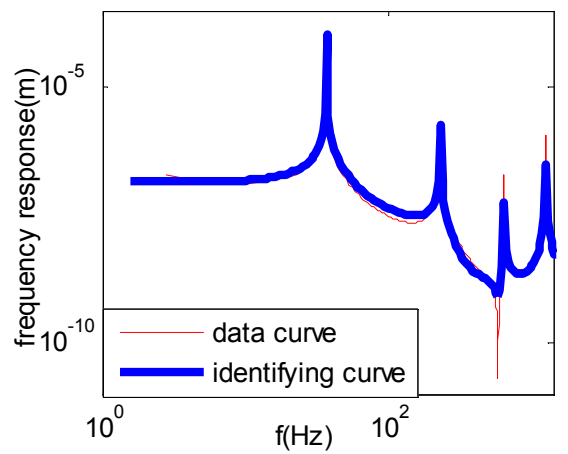

(a)

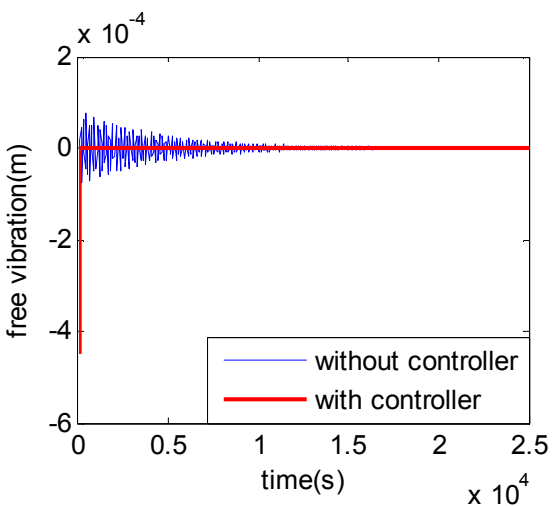

(b)

Fig. 7. The resluts in mechanical-electrical-thermal (423K) conditions (a) frequence response ---identification curve - experimental curve (b) free vibration — without controller — with controller

\section{Acknowledgements}

This work was funded by the National Natural Science Foundation of China, under grant no.10972137.

\section{Reference}

[1] Meng, G., L. Ye, et al. (2006). "Closed loop finite element modeling of piezoelectric smart structures." Shock and Vibration 13(1): 1-12.

[2] Giannopoulos, G. and J. Vantomme (2006). "A thermal-electrical-mechanical coupled FE formulation using discrete layer kinematics for the dynamic analysis of smart plates." Smart Materials \& Structures 15(6): 1846-1857.

[3] Kumar, R., B. K. Mishra, et al. (2008). "Thermally induced vibration control of cylindrical shell using piezoelectric sensor and actuator." International Journal of Advanced Manufacturing Technology 38(5-6): 551-562.

[4] Xue, X. and J. Tang (2006). "Robust and high precision control using piezoelectric actuator circuit and integral continuous sliding mode control design." Journal of Sound and Vibration 293(1-2): 335-359.

[5] Sethi, V. and G. Song (2008). "Multimodal vibration control of a flexible structure using piezoceramic sensor and actuator." Journal of Intelligent Material Systems and Structures 19(5): 573-582.

[6] Sethi, V. and G. Song (2005). "Optimal vibration control of a model frame structure using piezoceramic sensors and actuators." Journal of Vibration and Control 11(5): 671-684.

[7]Ezhilarasi, D., M. Umapathy, et al. (2006). "Design and experimental evaluation of piecewise output feedback control for structural vibration suppression." Smart Materials \& Structures 15(6): 1927-1938.

[8] Ma, K. and M. N. Ghasemi-Nejhad (2006). "Adaptive precision positioning of smart composite panels subjected to external disturbances." Mechatronics 16(10): 623-630.

[9] He, Chengli; Jha, Ratneshwar,(2003) "experimental evaluation of augmented UD identifiction based, vibration control of smart structures" Journal of Sound and Vibration, Volume 274, Issue 3-5, p. 1065-1078.

[10] Sethi, V. and G. Song (2006). "Pole-placement vibration control of a flexible composite I-beam using piezoceramic sensors and actuators." Journal of Thermoplastic Composite Materials 19(3): 293-307. 